\title{
Opacity of germanium and silicon in ICF plasmas
}

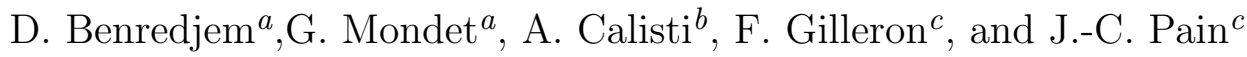 \\ ${ }^{a}$ Laboratoire Aimé Cotton, Université Paris-Sud, 91405 Orsay, France. \\ ${ }^{b}$ Aix-Marseille Université, CNRS, PIIM, Centre Saint Jérôme, 13397 Marseille, France \\ ${ }^{c}$ CEA, DAM, DIF, F-91297 Arpajon Cedex, France
}

\begin{abstract}
Because germanium and silicon may be used as dopants in the ablator of ignition target, the knowledge of their opacities is crucial. We have calculated the opacity by using two approaches. The first one utilizes a detailed line calculation in which the atomic database is provided by the MCDF code. A lineshape code was then adapted to the calculation of opacity profiles. Because the calculation time is prohibitive when the number of lines is huge, a second approach, combining detailed line calculations and statistical calculations is used. This approach necessitates much smaller calculation than the first one and is then well suited for extensive calculations. The monochromatic opacity and the Rosseland and Planck mean opacities are calculated for various relevant densities and temperatures.
\end{abstract}

\section{INTRODUCTION}

The knowledge of opacity and emissivity is very important when one deals with the radiative properties of ICF and astrophysical plasmas. Germanium and silicon as well as carbon may be present in the ablator of ICF schemes (LMJ in France, NIF at Livermore). Indirect-drive inertial confinement fusion uses a high-Z Hohlraum to convert precisely shaped laser pulse into thermal X-rays, which ablate the carbon-hydrogen-oxygen-germanium (CHOGe) ablator of some ignition designs. The radiation is absorbed due to the ablator opacity, setting up an ablation front that propagates into the ablator. Low density mass is ejected from the capsule surface, compressing the capsule in reaction (the DT fuel is compressed and a central hot spot is created at sufficiently high temperature and areal density for ignition). This spherical rocket compression process is dependent on the Hohlraum radiation temperature and on the opacity/emissivity of the ablator through the mass flux and the exhaust velocity.

The high quality of the X-ray radiation source is very important. Hard X-rays of $h \nu>1.8 \mathrm{keV}$ can propagate ahead of ablation front and preheat the inner layer of ablator next to the DT fuel, which increases the density difference between the fuel and ablator and results in the growth of hydrodynamic instabilities. Therefore, optimized designs of ignition capsule usually use the graded doped ablators such as germanium- or silicon-doped plastic. The role of the dopant is to absorb the hard X-ray radiation and to prevent preheating of the inner undoped plastic layer next to the DT fuel. The doped design limits Richtmyer-Meshkov and Rayleigh-Taylor instabilities as well as mixing at the fuel-ablator interface. The dopant concentration must be optimized: a too small quantity of dopant cannot efficiently prevent the preheating, and an excess of dopant tends to reduce the implosion velocity.

For low- or mid-Z dopants the number of lines is sometimes tractable. A detailed line calculation of the opacity is then appropriate. One can then calculate precisely the opacity profile and accounts for many line broadening mechanisms such as electron, Doppler and Stark broadening. Such an approach is time consuming and is limited by the number of levels generated by atomic data codes. We have used a detailed line calculation to obtain the opacity of germanium. This calculation involves the $\mathrm{MCDF}^{1}$ (atomic data) and PPP ${ }^{2}$ (line profile) codes. All major line broadening mechanisms (natural, collisional, Doppler and ion Stark broadening) are taken into account.

Because this approach is time consuming an alternative approach -hybrid approach- based on a detailed description whenever it is required and a statistical one when a huge number of lines overlap, is proposed. The

E-mail: djamel.benredjem@lac.u-psud.fr 
hybrid approach for opacity is the best compromise between precision and calculation time. The statistical part is calculated by the SCO (Super-Configuration Opacity) code, ${ }^{3}$ relying on the concept of "super-configuration".

In Sec. 2 we present the detailed line formalism generating the line shape code PPP. This code provides the intensity as well as the opacity profiles. This approach is applied to Ge lines. The number of lines is huge making the calculation time prohibitive for extensive calculations. In Sec. 3 we present the hybrid approach and a comparison between the two approaches is discussed. In Sec. 4 we present results of extensive calculations of monochromatic and mean opacities. All ionic stages of germanium and silicon are taken into account.

\section{DETAILED CALCULATIONS}

In a plasma, radiators interact with a perturbing bath formed by ions in various ionic stages and free electrons. The monochromatic intensity is expressed as the Fourier transform of the correlation function $C(t)$, i.e.

$$
I(\omega)=\frac{1}{\pi} \int_{0}^{\infty} d t \exp (i \omega t) C(t) .
$$

In Liouville-space notation the correlation function is expressed as the trace of the evolution operator, i.e.

$$
C(t)=\left\langle\left\langle\mathbf{d}^{\dagger}|U(t)| \rho \mathbf{d}\right\rangle\right\rangle,
$$

where $\mathbf{d}$ is the dipole operator of the radiator and $\rho$ the density matrix of the total system comprising the radiator and the perturbing bath.

In the standard theory of line shape two approximations are generally made. The perturbing ions are treated in the quasi-static approximation and their effect on the radiators spectrum is then represented by a static electric field which is characterized by a probability density $W$. The second approximation or impact approximation treats the free electrons as projectiles in binary collisions with the radiators. The effect of free electrons is then represented by a collision operator $\phi$, and consequently by a Liouville perturbation operator $\exp (-i \phi)$. The monochromatic intensity then reads

$$
I(\omega)=\frac{1}{\pi} \mathcal{R}\left\langle\left\langle\mathbf{d}^{\dagger}\left|\int d e W(e) \int_{0}^{\infty} d t \exp (i \omega t) \exp \left(-i\left(L_{e}-i \phi\right) t\right)\right| \rho \mathbf{d}\right\rangle\right\rangle
$$

where $\exp \left(-i\left(L_{e}-i \phi\right) t\right)$ is the evolution operator of the radiator perturbed by an (ion) electric field of value $e$ and free electrons. $W$ is given by a statistical distribution.

Owing to Stark effect, each radiative transition $u-l$ splits into $L$ Stark components (StC). ${ }^{4}$ Each StC is treated as a 2-level system and its spectral shape generally shows (i) an inhomogeneous (Doppler) broadening which is due to the thermal motion of ion perturbers and (ii) a homogeneous broadening which accounts for the natural broadening and the electron (impact) broadening. The monochromatic intensity then reads (see Ref. ${ }^{4}$ ):

$$
I(\omega)=\sum_{q=1}^{L} \frac{c_{q}\left(\omega-f_{q}\right)+a_{q} \gamma_{q}}{\left(\omega-f_{q}\right)^{2}+\gamma_{q}^{2}},
$$

where $\left(a_{q}+i c_{q}\right)$ are the generalized intensities and $\left(f_{q}+i \gamma_{q}\right)$ the generalized frequencies. The intensity can be seen as resulting from the contribution of $L$ two-level systems. It takes the following form:

$$
I(\omega)=\sum_{q=1}^{L} a_{q} \Psi_{q}(\omega),
$$

where $\Psi_{q}$ is the profile of the StC $q$ belonging to the radiative line $u-l$. We define an effective line strength for each StC by setting $a_{q}=S_{q} / e^{2}$, in atomic units.

Considering that each 2-level system contributes to the emission and absorption processes, it is possible to define an opacity and an emissivity for each StC. 
When one accounts for ion Stark broadening, each radiative transition gives rise to many StC's, and the absorption coefficient of an ion of net charge $Z_{i} e$ is then given by

$$
k_{i}(\nu)=\sum_{u-l} \sum_{q=1}^{L} N_{l, q} \frac{8 \pi^{3}}{3 h c} a_{0}^{2} f_{q} S_{q} \Psi_{q}(\nu),
$$

where the sum on $u-l$ accounts for all radiative transitions and the sum on $q$ accounts for all StC associated with a radiative transition $u-l . L$ is the number of $\mathrm{StC}$ associated with a transition $u-l$ and $N_{l, q}$ is the population density of the lower level of the StC $q$.

The opacity reads:

$$
\kappa_{i}(\nu)=\frac{k_{i}(\nu)}{\rho},
$$

where $\rho$ is the mass density.

We have calculated the opacity of $\mathrm{Ge}^{18+}$ in the ground superconfiguration $\mathrm{SC}(3)^{4}$, with full $\mathrm{K}$ and L shells. Generally the ground SC is of the type $(n)^{r}$. We restrict ourselves to $n=3$ and $r=4$.

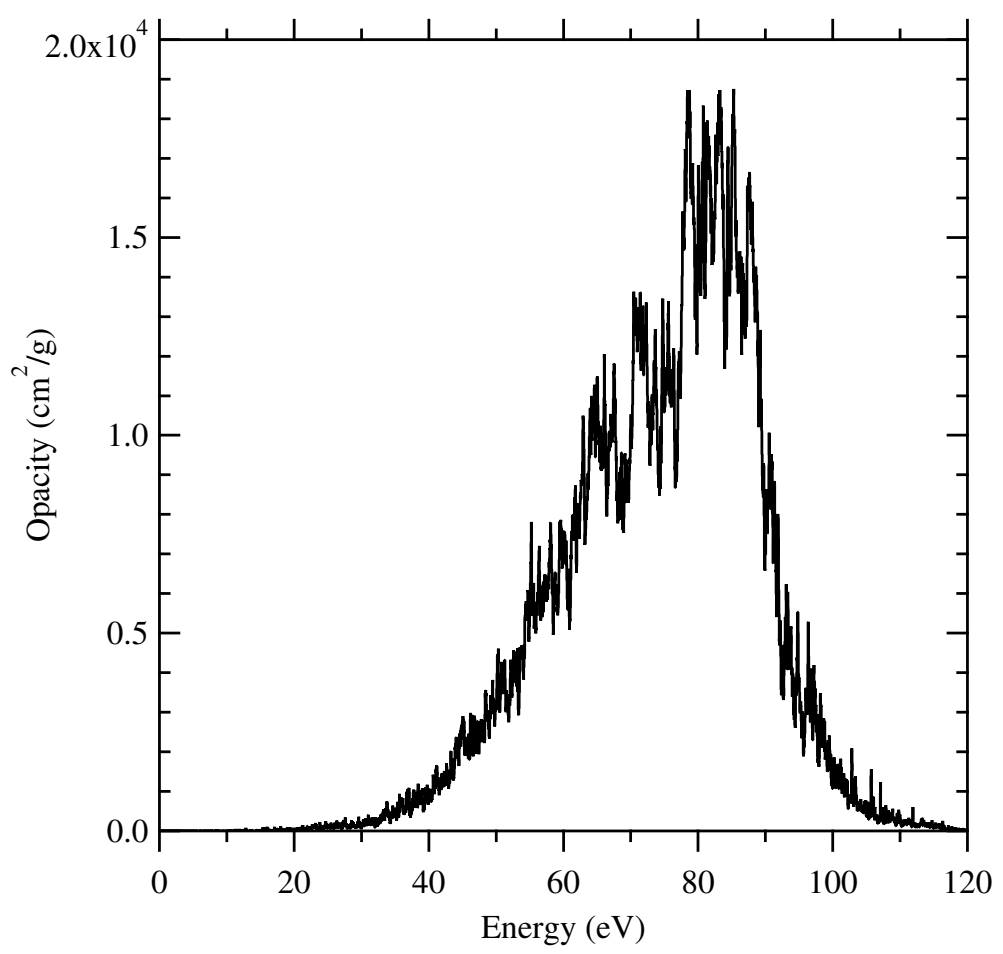

Figure 1. Opacity of $\mathrm{Ge}^{18+}$ in the $\mathrm{SC}(3)^{4}$ as a function of the photon energy. The electron density and temperature are fixed to $1.7 \times 10^{23} \mathrm{~cm}^{-3}$ and $200 \mathrm{eV}$, respectively.

In Fig. 1 we show the opacity of $\mathrm{Ge}^{18+}$. The number of lines if huge making the calculation time sometimes prohibitive, especially when ion Stark effect is taken into account. Fortunately, the ion Stark broadening is negligible in this particular case.

However for such SC's the precise opacity profile given by Eq. 6 requires large calculation times in many cases. Then extensive calculations involving many ion stages are not tractable. We have thus explored a much faster approach called hybrid approach. This approach combines detailed calculations and statistical calculations. In this approach detailed calculations are performed only if they are necessary, resulting in a considerable reduction of calculation time. 


\section{HYBRID APPROACH}

Let us define quantities such as configuration, super-configuration ... which are relevant in atomic spectroscopy. A configuration $C$ reads $\left(n_{1} l_{1}\right)^{N_{1}}\left(n_{2} l_{2}\right)^{N_{2}}\left(n_{3} l_{3}\right)^{N_{3}} \cdots$, where $n$ and $l$ are the principal and orbital quantum numbers, respectively. $N$ is the number of electrons occupying the sub-shell $n l$. The $N^{\prime} s$ fulfill the condition $\sum_{i} N_{i}=N_{b}$, where $N_{b}$ is the number of bound electrons. A super-configuration (SC) reads $\left(\sigma_{1}\right)^{N_{1}}\left(\sigma_{2}\right)^{N_{2}}\left(\sigma_{3}\right)^{N_{3}} \ldots$, where the super-shell $\sigma$ is an ensemble of sub-shells. Now $N$ is the number of electrons occupying the super-shell $\sigma$. For instance $\left(n_{1}\right)^{N_{1}}\left(n_{2}\right)^{N_{2}}\left(n_{3}\right)^{N_{3}} \ldots$ is a particular case of super-configuration where all the sub-shells having the same principal quantum number are gathered. In that case, each super-shell is an ordinary shell. Note that $(n)^{N}$ is sometimes called a Layzer complex, but this is not true since a Layzer complex stricto sensu has a fixed parity ; therefore $(n)^{N}$ represents in fact two Layzer complexes. A transition array is the ensemble of transitions between two configurations of the same ion :

$$
\left\{\left(n_{1} l_{1}\right)^{N_{1}}\left(n_{2} l_{2}\right)^{N_{2}}\left(n_{3} l_{3}\right)^{N_{3}} \cdots-\left(n_{1} l_{1}\right)^{N_{1}-1}\left(n_{2} l_{2}\right)^{N_{2}+1}\left(n_{3} l_{3}\right)^{N_{3}} \cdots\right\}
$$

A configuration is determined by a set of $N_{i}$ : $C\left(N_{1}, N_{2}, N_{3} \cdots\right)$. A supertransition array (STA) is the ensemble of transitions arrays $\left\{C\left(N_{1}, N_{2}, N_{3} \cdots\right)-C\left(N_{1}-1, N_{2}+1, N_{3} \cdots\right)\right\}$. The spectral broadening is often responsible for a coalescence of the lines pertaining to a transition array. In such cases the structure is named unresolved transition array (UTA)

The developed hybrid approach ${ }^{5}$ fulfills the following requirements. The associated opacity code SCO-RCG must be a tool for the diagnostic and interpretation of spectroscopy experiments in LTE plasmas as well as for the study of the intrinsic properties of complex atomic spectra. It must ensure thermodynamic consistency, use limited resources, and obey an automatic execution, in order to generate opacity tables. SCO-RCG combines statistical $\mathrm{STA}^{6}$ and $\mathrm{UTA}^{7}$ approaches as well as fine-structure calculations. It involves definition of criteria in order to decide whether a detailed treatment of the lines is necessary and to determine the validity of the statistical methods. The statistical part of the spectrum is given by the code $\mathrm{SCO},{ }^{3}$ which relies on the concept of SC.

But if the STA approach enables one to deal with the large number of configurations, it does not provide a precise description of the individual electric-dipolar lines inside a given transition array. It can therefore be improved if several transition arrays are treated in a detailed way (line-by-line). This implies to define criteria in order to decide whether a detailed calculation is necessary and possible or not, and to clarify the validity of statistical approaches. The data required for the calculation of transition arrays (Slater integrals, spin-orbit and dipolar matrix elements) are evaluated and transmitted by SCO (Cowan's code was indeed designed in such a way that the radial and dipolar integrals can be entered as parameters). Therefore, the plasma environment effects (screening) on the wave-functions are taken into account thanks to the thermodynamic modelling of SCO. Then, the energies of the levels and lines (positions and strengths) are calculated by the Cowan's code. ${ }^{8}$ The estimate of the number of lines within a transition array is an important ingredient, in order to decide whether a detailed calculation is suitable or not for the transition array, as well as the number of levels with fixed angular momentum $J$ which represents the size of a block of the Hamiltonian matrix. This led us to develop efficient algorithms for the numbering of $L S$ terms and $J$ levels. ${ }^{9}$ We have also extended the statistical modelling of the distribution of quantum states in order to better describe configurations having open sub-shells with a large angular momentum.

In SCO-RCG, configuration interaction is limited to electrostatic interaction between relativistic subconfigurations ( $n l j$ orbitals) of a non-relativistic configuration ( $n l$ orbitals), namely relativistic configuration interaction (RCI). For the detailed part of the spectrum, RCI is calculated exactly, through the diagonalization of the Hamiltonian matrix. For the statistical part of the spectrum, RCI is described using an approximate model, which relies on the definition, in a transition array, of two sets of lines by considering only one relativistic orbital (the one with the largest spin-orbit integral in the transition). Corrections to the energy and strength of both structures are derived in the first-order perturbation theory, in order to include electrostatic effects between levels of the sub-arrays. Each transition array is represented by two distributions which characteristics (mean energy, strength, and number of lines) change in a continuous way from $L S$ (where one sub-array vanishes) to jj coupling. ${ }^{10}$ 
The hybrid code enables one to obtain precise opacities, required for astrophysics and inertial confinement fusion, and to study the properties of complex atomic spectra. It includes the effect of plasma environment, uses limited computational resources, and follows an automatic execution, making the generation of opacity tables easier.

The broadening mechanisms taken into account are inelastic collisions in the impact approximation, Stark effect from the neighboring ions in the quasi-static approximation, Doppler effect (thermal motion of ions) and natural width. The elastic contribution to electron collisions is neglected. Doppler effect and ionic Stark effect are modeled by a Gaussian profile and the effect of inelastic collisions with electrons is described, as the natural width, by a Lorentzian profile. The convolution of the Gaussian by the Lorentzian profiles leads to a Voigt profile.

The electron collisions are described within an approach proposed by Dimitrijević and Konjević. ${ }^{11-13}$ Such a formulation relies on Baranger's expression for the width of an isolated line. ${ }^{14,15}$ The treatment of ionic Stark effect in SCO-RCG was proposed by Rozsnyai. ${ }^{16}$

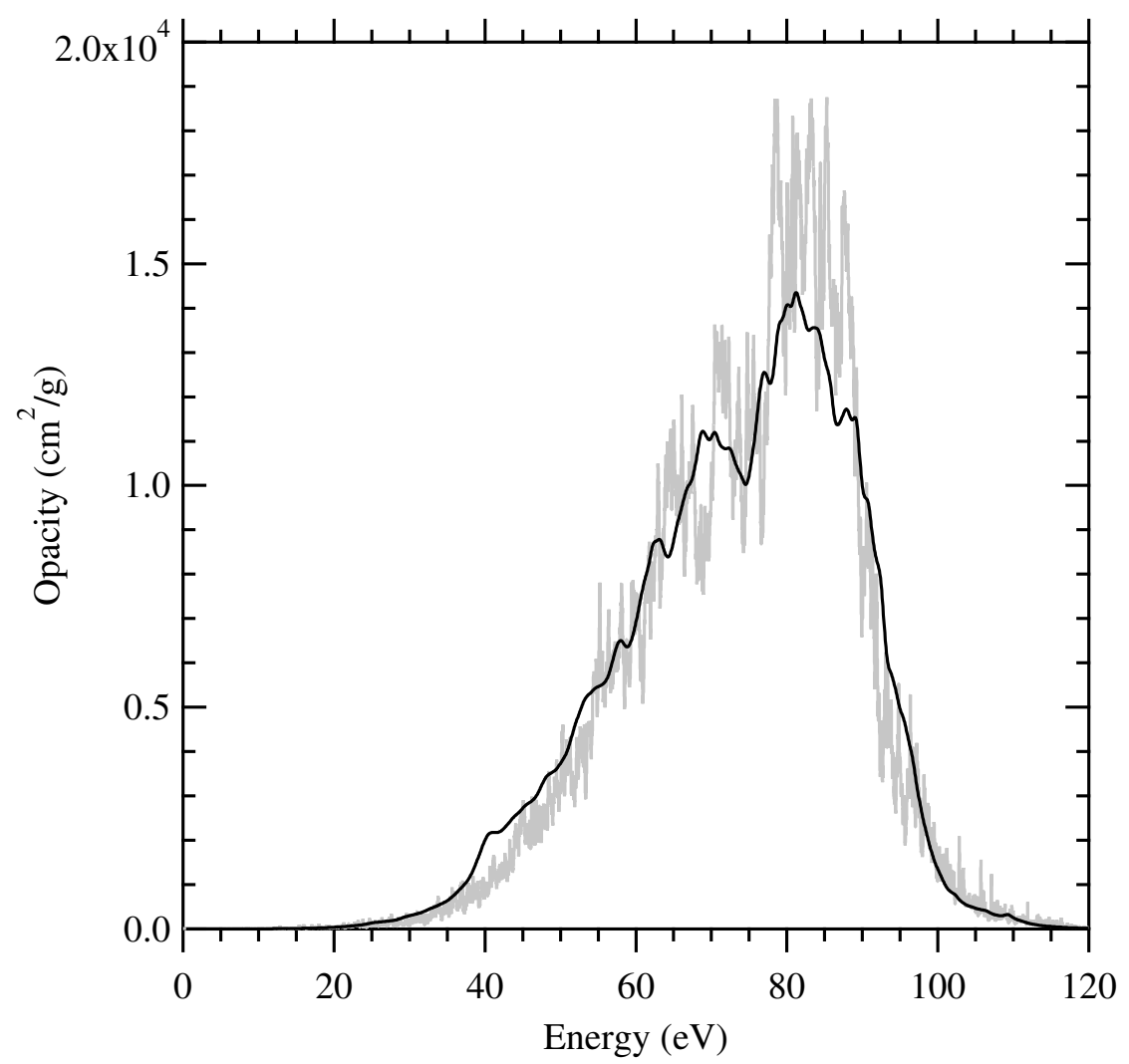

Figure 2. Opacity of $\mathrm{Ge}^{18+}$ in the $\mathrm{SC}(3)^{4}$ as a function of the photon energy. Gray curve: PPP approach, black curve: hybrid approach. $N_{e}$ and $T_{e}$ values, as in Fig. 1.

In Fig. 2 we compare the opacities of $\mathrm{Ge}^{18+}$, in the $\mathrm{SC}(3)^{4}$ (see above), obtained by the detailed line calculations and within the hybrid approach. The detailed calculations using the PPP code showed that the ion Stark broadening is negligible. This is due to the small value of $n$. A precise knowledge of the spectral broadening is not necessary because the lines overlap due to a huge number of radiative transitions. The agreement between the two approaches is good, except in the $80 \mathrm{eV}$ energy range. This small discrepancy is attributed to the less precise treatment of the electron broadening in the hybrid approach. The agreement between the two approaches gives us confidence in the hybrid approach for extensive calculations of opacity. Such a model appears to be a powerful tool for the interpretation of recent laser and Z-pinch experimental spectra, as well as for validation of statistical methods. 
In the following we concentrate on germanium and silicon plasma. All relevant ionic stages are taken into account. The number of lines is huge making the full detailed line calculations (PPP approach) prohibitive. It is preferable to use the hybrid approach which combines detailed line calculations, whenever it is necessary, and statistical calculations in the other cases.

\section{OPACITY OF GERMANIUM AND SILICON WITHIN THE HYBRID APPROACH}

In the following we account for all relevant ionic stages. The opacity then reads:

$$
\kappa(\nu)=\sum_{i} p_{i} \kappa_{i}(\nu)
$$

where $p_{i}$ is the fraction of ions of net charge $z_{i} e$. Obviously $\sum_{i} p_{i}=1$. The ionic populations are assumed in LTE.

In Fig. 3 we compare the total opacity of germanium and silicon. Total opacity involves bound-bound (b-b), bound-free (b-f) and free-free (f-f) transitions. The opacity of germanium overcomes that of silicon except at $\simeq 300 \mathrm{eV}$ and $\simeq 1800 \mathrm{eV}$ where the $\mathrm{b}-\mathrm{b}$ contribution of $\mathrm{Si}$ is important. When $T_{e}$ is increased to $500 \mathrm{eV}$ we notice

- a strong decrease in the spectral range $0-1500 \mathrm{eV}$ for both $\mathrm{Ge}$ and $\mathrm{Si}$,

- at $h \nu \simeq 1700 \mathrm{eV}$ there is an increase of opacity of Ge because higher levels are populated,

- at $h \nu \simeq 1850 \mathrm{eV}$, the opacity of $\mathrm{Si}$ decreases while peaks at $2 \mathrm{keV}$ emerge, for the same reason as above.
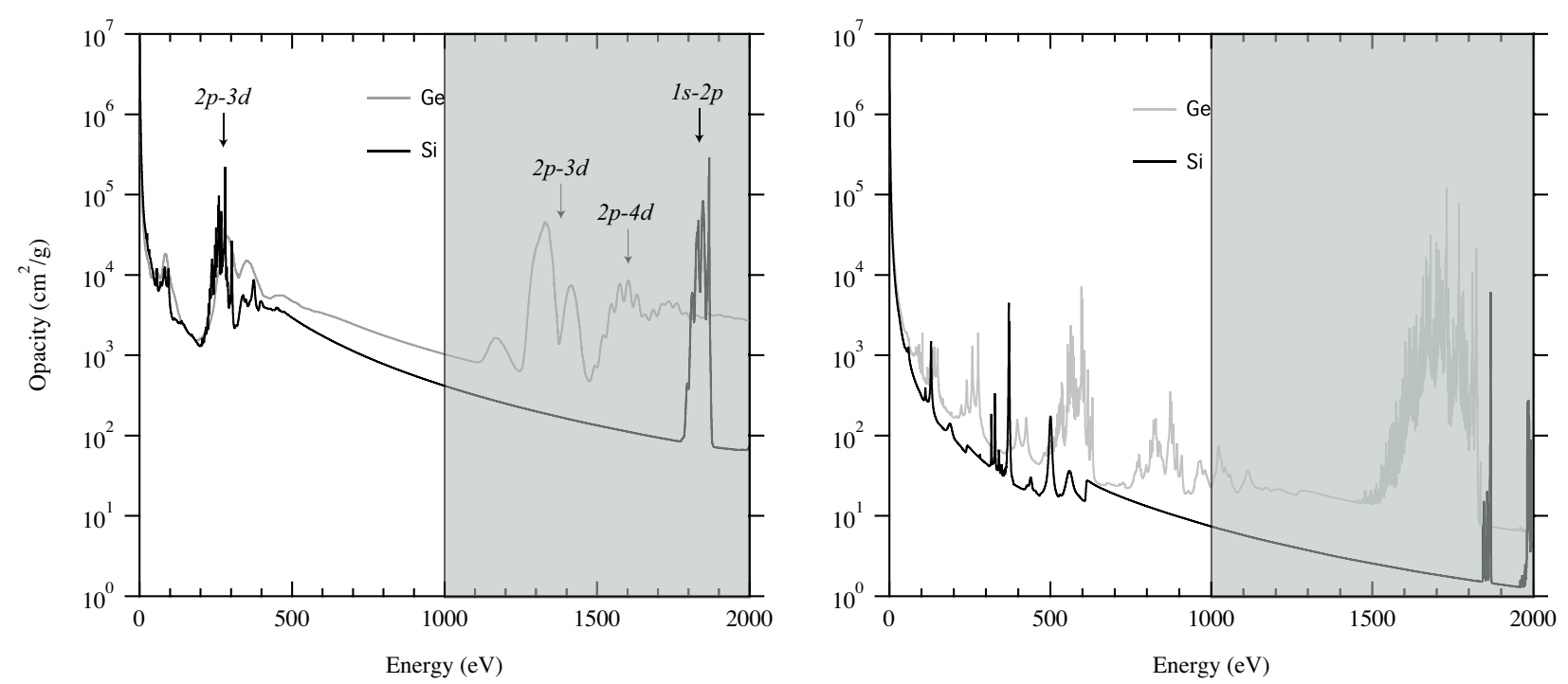

Figure 3. Ge and Si opacities for $\rho=0.1 \mathrm{~g} \mathrm{~cm}^{-3}$. Grey curve: Ge, black curve: Si. Left: $T_{e}=100 \mathrm{eV}$, right: $T_{e}=500 \mathrm{eV}$.

In Fig. 4 we show the temperature variation of the total opacity. As previously noticed, the opacity of germanium at $100 \mathrm{eV}$ is higher than that at $500 \mathrm{eV}$, except at $\simeq 1700 \mathrm{eV}$. We observe an increase of opacity at higher energies. This is explained by the increase of populations of high energy levels.

In Fig. 5 we have represented the opacity for $T_{e}=300 \mathrm{eV}$ and three different densities. The opacity increases with density. If we compare the opacity of Ge at $0.1 \mathrm{~cm}^{2} / \mathrm{g}$ and at $1 \mathrm{~cm}^{2} / \mathrm{g}$, we notice a line merging due to an increasing spectral broadening. 

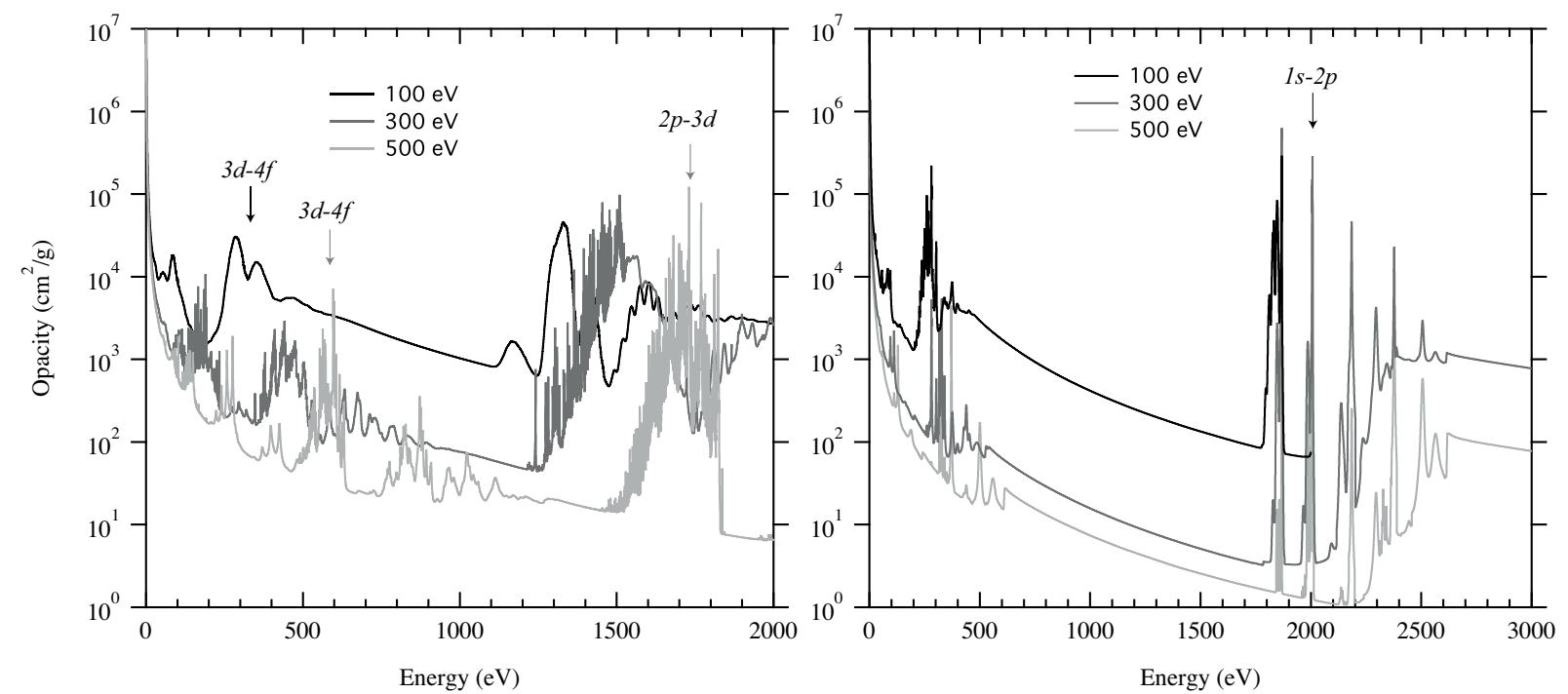

Figure 4. Total opacity for $\rho=0.1 \mathrm{~g} \mathrm{~cm}^{-3}$ and three different electron temperatures. Left: Ge, right: Si.
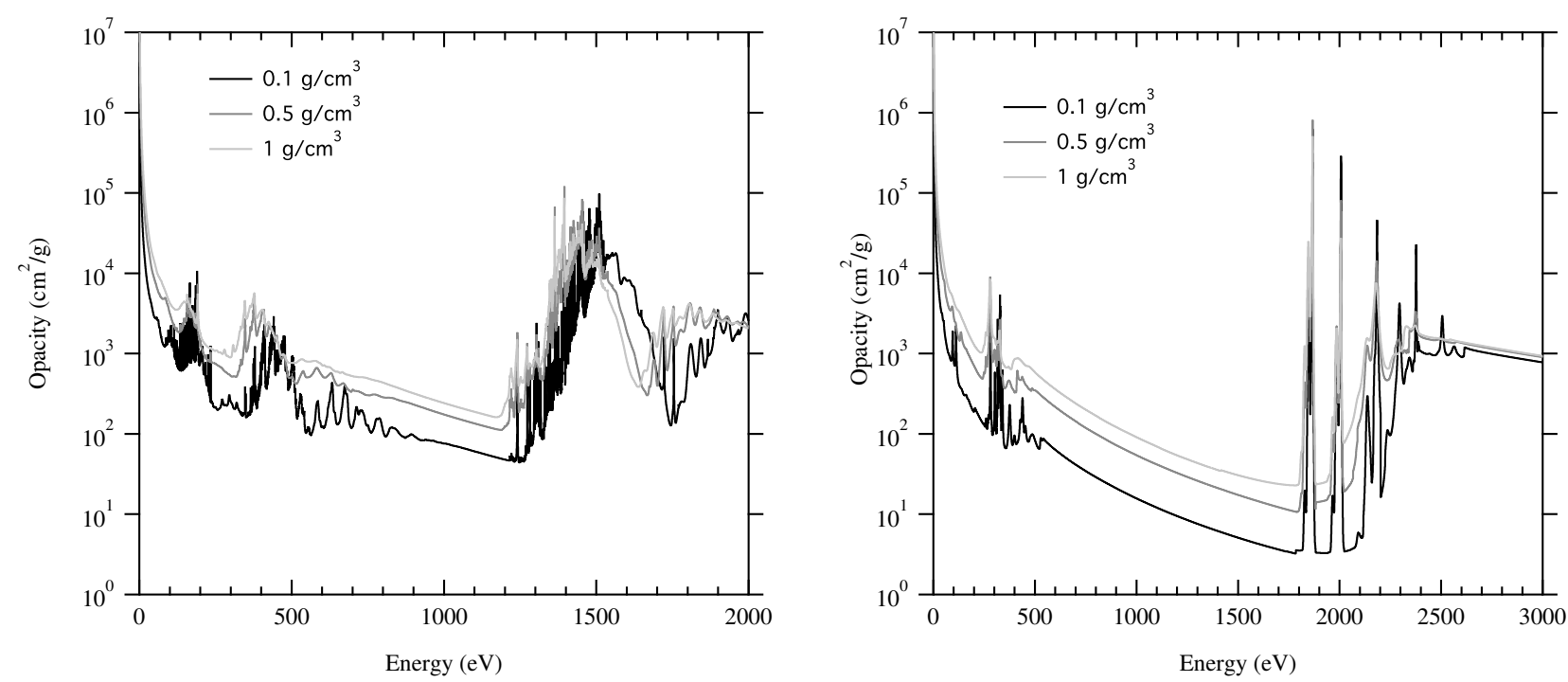

Figure 5. Total opacity for $T_{e}=300 \mathrm{eV}$ and three different densities. Left: Ge, right: Si.

Now we focus on mean opacities. We define the Rosseland, $\kappa_{R}$, and Planck, $\kappa_{P}$, mean opacities by

$$
\frac{1}{\kappa_{R}}=\frac{15}{4 \pi^{4}} \int_{0}^{\infty} \frac{1}{\kappa(u)} \frac{u^{4} \exp (-u)}{[1-\exp (-u)]^{2}} d u
$$

and

where $u=h \nu / k T_{e}$.

$$
\kappa_{P}=\frac{15}{\pi^{4}} \int_{0}^{\infty} \kappa(u) \frac{u^{3}}{\exp u-1} d u
$$

In Fig. 6 we show the temperature variation of Rosseland and Planck mean opacities of Ge and Si. The density is fixed to $0.1 \mathrm{~g} \mathrm{~cm}^{-3}$. We can see that the Planck opacity overcomes the Rosseland opacity. When the temperature increases the average ion charge increases and thus the opacities decrease. Analytical expressions for mean opacities would be useful. In a recent work on the opacity of carbon ${ }^{17}$ analytical expressions that fit fairly well the calculated opacities are proposed. 

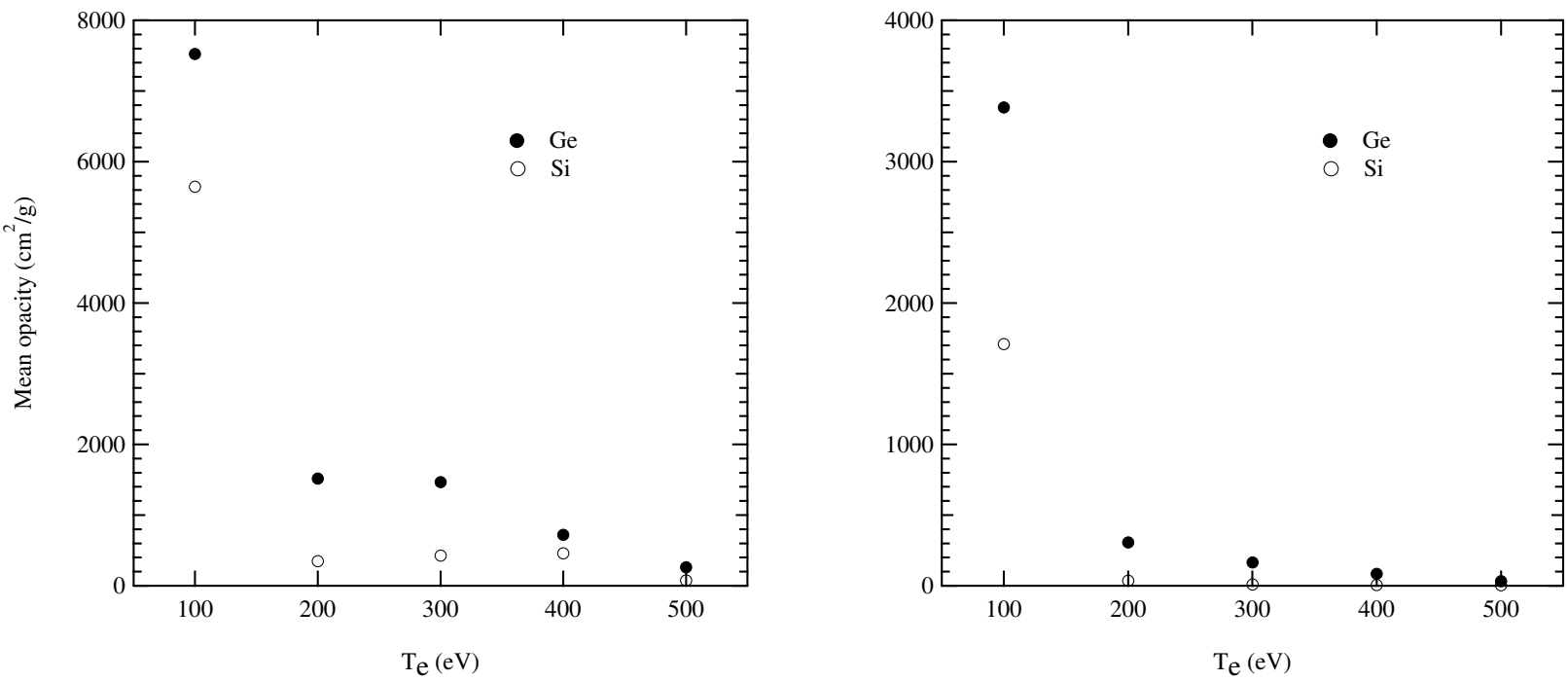

Figure 6. Planck (left) and Rosseland (right) mean opacities of Ge and Si as a function of $T_{e} \cdot \rho=0.1 \mathrm{~g} \mathrm{~cm}{ }^{-3}$.

\section{CONCLUSION}

Monochromatic opacity can be calculated within a detailed line approach where all major line broadening mechanisms are treated precisely. Because this approach is time consuming when a huge number of transitions is involved, a hybrid approach combining statistical and detailed calculations was explored. This approach was used to obtain the monochromatic opacity of germanium and silicon dopants in ICF capsules. We have investigated the temperature and density variations. We have also calculated the mean Planck and Rosseland opacities. In a future work we plan to investigate the effect of line broadening on mean opacities.

\section{REFERENCES}

1. I. P. Grant, B. J. McKenzie, P. H. Norrington, D. F. Mayers and N. C. Pyper, Comput. Phys. Comm. 21, 207-231 (1980).

2. B. Talin, A. Calisti, L. Godbert, R. Stamm, R. W. Lee and L. Klein, Phys. Rev. A 5, 1918-1928 (1995).

3. T. Blenski, A. Grimaldi and F. Perrot, J. Quant. Spectrosc. Radiat. Transfer 65, 91-100 (2000).

4. D. Benredjem, A. Calisti, G. Mondet, J.-C. Pain and F. Gilleron, Can. J. Phys. 89, 609 (2011).

5. Q. Porcherot, J.-C. Pain, F. Gilleron and T. Blenski, High Energy Density Phys. 7, 234 (2011).

6. A. Bar-Shalom, J. Oreg, W. H. Goldstein, D. Shvarts and A. Zigler, Phys. Rev. A 40, 3183-3193 (1989).

7. J. Bauche, C. Bauche-Arnoult, and M. Klapisch, Phys. Rev. A 20, 2024 (1979).

8. R. D. Cowan, The Theory of Atomic Structure and Spectra], University of California Press, Berkeley (1981).

9. F. Gilleron and J.-C. Pain, High Energy Density Phys. 5, 320 (2009).

10. F. Gilleron, J. Bauche and C. Bauche-Arnoult, J. Phys. B: At. Mol. Opt. Phys. 40, 3057 (2007).

11. M. S. Dimitrijević and N. Konjević, J. Quant. Spectrosc. Radiat. Transfer 24, 451 (1980).

12. M. S. Dimitrijević and N. Konjević, Astron. Astrophys. 163, 297 (1986).

13. M. S. Dimitrijević and N. Konjević, Astron. Astrophys. 172, 345 (1987).

14. M. Baranger, Phys. Rev. 111, 494 (1958).

15. M. Baranger, in D. R. Bates, Atomic and molecular processes (Academic Press, New-York, 1962).

16. B. F. Rozsnyai, J. Quant. Spectrosc. Radiat. Transfer 17, 77 (1977).

17. E. Mínguez, R. Florido, R. Rodríguez, J. M. Gil, J. G. Rubiano, M. A. Mendoza, D. Suárez and P. Martel, High Energy Density Phys. 6, 57-65 (2010). 